\title{
Diacronie
}

Studi di Storia Contemporanea

$\mathrm{N}^{\circ} 25,1 \mid 2016$

"Se creare è definire"

\section{Mare nostrum e «Geopolitica». Il mito imperiale dei geografi italiani}

\section{Andrea Perrone}

\section{(2) OpenEdition}

\section{Journals}

\section{Edizione digitale}

URL: http://journals.openedition.org/diacronie/3936

DOI: 10.4000/diacronie.3936

ISSN: 2038-0925

\section{Editore}

Association culturelle Diacronie

Notizia bibliografica digitale

Andrea Perrone, « Mare nostrum e «Geopolitica». II mito imperiale dei geografi italiani », Diacronie

[Online], N² 25, 1 | 2016, documento 4, Messo online il 29 mars 2016, consultato il 03 mai 2019. URL http://journals.openedition.org/diacronie/3936 ; DOI : 10.4000/diacronie.3936 


\title{
Diacronie
}

\section{Mare nostrum e «Geopolitica»}

\section{Il mito imperialedei geografi italiani}

\author{
Andrea PERRONE *
}

L'articolo presenta un'analisi dettagliata della storia del periodico «Geopolitica» (1939-1942), legata alla tradizione geografico-politica italiana e sviluppatasi a partire dai padri del Risorgimento fino agli anni Trenta del secolo scorso. La strategia editoriale perseguita dalla direzione del periodico fu - in maniera un po' troppo ambiziosa - quella di orientare le linee direttrici della politica imperiale ed espansionistica del regime fascista nell'area di tutto il Mediterraneo, da Est a Ovest, ovvero dallo stretto di Gibilterra, passando per il Canale di Suez, fino alle isole dell'Egeo per estromettere inglesi e francesi dai loro domini coloniali. Alla rivista, che ebbe un'impostazione di natura multidisciplinare, parteciparono alcuni fra i maggiori studiosi italiani delle diverse discipline storiche, politiche e geografiche.

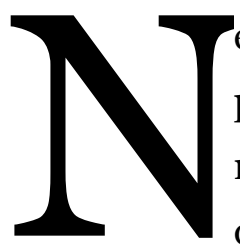

el gennaio 1939, a ridosso della Seconda guerra mondiale, la geografia politica italiana raggiunse il suo acme evolutivo con la pubblicazione della rivista «Geopolitica», raccogliendo attorno a sé i maggiori studiosi dell'epoca, in linea con una visione organica e multidisciplinare delle scienze territoriali, al passo con le più moderne nazioni europee ed extraeuropee dell'epoca ${ }^{1}$.

\footnotetext{
${ }^{1}$ Per un inquadramento generale sulle origini e gli sviluppi della Geopolitica italiana si possono leggere con profitto le seguenti pubblicazioni: LOSANO, Mario G., La geopolitica del Novecento. Dai Grandi Spazi alle delle dittature alla decolonizzazione, Milano, Bruno Mondadori Editore, 2011; SINIBALDI, Giulio, La geopolitica in Italia (1939-1942), Limena, Edizioni Webster Srl, 2010; ANTONSICH, Marco, «Geopolitica: The 'Geographical and Imperial Consciousness' of FascistItaly», in Geopolitics, 14, 2009, pp. 256-277; BORIA, Edoardo, Cartografia e potere. Segni e rappresentazioni negli atlanti italiani del Novecento, Torino, UTET, 2007; JEAN, Carlo, La geopolitica secondo l'Enciclopedia del Novecento, in Enciclopedia del Novecento, vol.
} 
La strategia perseguita dal periodico divenne quella di orientare le linee direttrici della politica imperiale del fascismo in stretta relazione con la posizione geografica della Penisola e con gli avvenimenti in corso a livello mondiale che vedevano l'Italia coinvolta nel confronto bellico ${ }^{2}$.

I fautori del progetto editoriale furono Giorgio Roletto, professore ordinario di Geografia economica all'Università di Trieste, e di Geografia politica ed economica all'Ateneo di Ferrara e di Padova, rispettivamente nella facoltà di Scienze sociali e sindacali e in quella di Scienze politiche, e il suo allievo Ernesto Massi, all'epoca docente di Geografia politica ed economica all'Università Cattolica del Sacro Cuore di Milano e all'Università di Pavia, presso la facoltà di Scienze Politiche dei due Atenei. Al loro fianco collaborarono un numero molto vasto di studiosi di formazione diversa e di varie discipline che sostennero con i loro contributi editoriali il progetto avanzato dai due corifei della geopolitica italiana.

Non possiamo esimerci dal ricordare che il periodico venne alla luce per merito dello sforzo congiunto dei due geopolitologi con l'aiuto di Padre Agostino Gemelli, rettore dell'Università Cattolica del Sacro Cuore di Milano, e con il placet del Ministro dell'Educazione Nazionale, Giuseppe Bottai, allo scopo di sostenere la politica estera del Fascismo e con la speranza di influenzare le scelte strategiche ed imperiali del

X, Supplemento II. A-G, Roma, Istituto della Enciclopedia Italiana, 1998, disponibile anche su URL: $\quad<\quad$ http://www.eurasia-rivista.org/la-geopolitica-secondo-lenciclopedia-delnovecento/17707/ > [consultato il 24 maggio 2015]; SINIBALDI, Giulio, «Alle origini della rivista Geopolitica (1939-1942)», in Clio. Rivista trimestrale di studi storici, XLI, 2/2005, pp. 267-294; VINCI, Anna, “'Geopolitica' e Balcani: l'esperienza di un gruppo di intellettuali in un ateneo di confine», in Società e Storia, XIII, 47, 1990, pp. 87-127, ristampato in appendice a LOROT, Pascal, Storia della Geopolitica, Trieste, Asterios Editore, 1997, pp. 117-141; ANTONSICH, Marco, La géopolitique méditerranèenne de l'Italie, in COUTAU-BÉGARIE, Hervé (sous la dir. de), L'évolution de la Pensée Navale, vol. V, Paris, Economica, 1995, pp. 163190.

2 DE FELICE, Renzo, Mussolini il duce. Gli anni del consenso 1929-1936, Torino, Einaudi, 1974; DE FELICE, Renzo, Mussolini il duce. Lo Stato totalitario 1936-1940, Torino, Einaudi, 1981; DE FELICE, Renzo, Mussolini l'alleato, vol. I, L'Italia in guerra 1940-1943, Einaudi, Torino, 1990; GIN, Emilio, L'ora segnata dal destino. Gli Alleati e Mussolini da Monaco all'intervento. Settembre 1938-Giugno 1940, Roma, Edizioni Nuova Cultura, 2012; MILANO, Rosario, MONZALI, Luciano, Dalla ricerca dell'equilibrio al sogno dell'egemonia. Appunti sulla politica estera italiana nello spazio mediterraneo tra le due guerre mondiali, in DE LEO, Raffaele, LOVECCHIO, Antonia (a cura di), Bari, La Puglia, l'Oriente. 'L'invenzione' di un ruolo internazionale, Lecce, Besa, 2014; QUARTARARO, Rosaria, Roma tra Londra e Berlino. La politica estera fascista dal 1930 al 1940, 2 voll., Sesto San Giovanni, Jouvence, 2012; BOTTONI, Riccardo (a cura di), L'Impero fascista. Italia ed Etiopia (1935-1941), Roma-Bari, Laterza, 2008; AGA-ROSSI, Elena, GIUSTI, Maria Teresa, Una guerra a parte. I militari italiani nei Balcani, 1940-1945, Bologna, Il Mulino, 2011; AGA-ROSSI, Elena, La politica estera e l'Impero, in SABBATUCCI, Giovanni, VIDOTTO, Vittorio, (a cura di), Storia d'Italia. Guerre e fascismo (1914-1943), vol. IV, Roma-Bari, Laterza, 1997; CACCAMO, Francesco, MONZALI, Luciano (a cura di), L'occupazione italiana della Jugoslavia, Le Lettere, Firenze, 2008; RODOGNO, Davide, Il nuovo ordine mediterraneo. Le politiche di occupazione dell'Italia fascista in Europa (1940-1943), Torino, Bollati Boringhieri, 2003. 
regime fascista nelle aree geopolitiche considerate di stretta competenza dell'Italia: l'Africa nord-orientale, le due sponde dell'Adriatico con la Dalmazia e l'Albania, le Sporadi meridionali, la Corsica, Nizza, Savoia, Il Canton Ticino. Inoltre, era necessario - a detta dei geopolitologi italiani - ottenere il pieno controllo del Canale di Suez e dello stretto di Gibilterra, all'epoca in mano ai britannici, per impedire un eventuale blocco economico-militare.

Fu lo stesso Massi che, incoraggiato da Roletto, concentrò lo studio della geopolitica in modo da opporsi a quella che all'epoca veniva considerata una spartizione iniqua delle risorse mondiali, consacrata a Versailles, e indifferente - come sottolineavano gli studiosi di geopolitica italiani - al diritto demografico delle nazioni, al diritto dei popoli alla terra, alle giuste esigenze degli Stati dotati di una popolazione sovrabbondante ${ }^{3}$. Le «ingiustizie» perpetrate al tavolo della pace, con la cosiddetta «Vittoria mutilata», verranno condannate dai geografi italiani anche più tardi, puntando il dito contro la Società delle Nazioni, istituita subito dopo la Grande Guerra e diventata - nella loro ottica - lo strumento di salvaguardia degli interessi delle «nazioni ricche». Interessi costituiti prevalentemente da fattori di natura economico-finanziaria e riguardanti il controllo delle materie prime per difendere lo status quo creato a Versailles ai danni delle «nazioni proletarie» come l'Italia, che ora guardavano ad un'espansione di stampo coloniale nelle zone ritenute di loro competenza geostrategica e geoeconomica ${ }^{4}$, per reperire risorse utili alla loro sopravvivenza e al loro sviluppo.

Giova ricordare che la politica estera del regime non fece altro che, sulla spinta del suo orientamento decisamente nazionalista ed espansionista, aumentare il processo di gravitazione del settore alpino orientale e adriatico, compresa l'Albania e le isole Ionie, il Dodecanneso e Rodi, fino ad auspicare la nascita di un ponte di collegamento in direzione dell'Anatolia, per il controllo di un'area geografica particolarmente ricca di giacimenti di carbone. In questo, le scelte di politica estera e geostrategiche di Mussolini furono orientate a coinvolgere l'Istituto Geografico Militare (I.G.M.), soprattutto nel settore geotopografico, facendo proprie le istanze che rammentavano le vestigia imperiali di Roma - più volte ricordate anche negli articoli pubblicati su «Geopolitica» - e che erano finalizzate ad una politica di stampo imperiale sull'onda di quelle che erano state le finalità dei nostri ex alleati nella Grande Guerra5.

\footnotetext{
3 MASSI, Ernesto, «Democrazie, colonie e materie prime», in Geopolitica, I, 1/1939, pp. 17-35, p. 18.

4 DE MAGISTRIS, Luigi Filippo, Per non dimenticare, Milano, G. De Agostini Edizioni, 1936.

5 MANGLAVITI, Stefano, «Rappresentazioni geografiche e visione geopolitica, un laboratorio d'indagine: la visione dei Balcani attraverso la produzione dell'Istituto Geografico Militare nel ventennio", in L'Universo, 86, 6/2006, pp. 821-845, pp. 842-843.
} 
Del resto, il mancato riconoscimento delle intese alla fine del primo conflitto mondiale aveva provocato la "Vittoria mutilata", per cui non erano stati riconosciuti i diritti sui territori dell'area nord-orientale legati storicamente all'Italia, dall'antica Roma alla Serenissima ${ }^{6}$ : la Dalmazia, le isole Curzola, Lissa e Brazza, la città di Fiume con la Liburnia e due delle maggiori isole del Carnaro (Veglia e Arbe). A cui si aggiunse una costruzione artificiosa di Stati come la Jugoslavia e la Cecoslovacchia, dove convivevano a stento numerose minoranze etniche.

$\mathrm{Al}$ termine della Prima guerra mondiale, precisò Massi alcuni decenni dopo, furono soprattutto i popoli slavi ad uscire rafforzati dalle controversie diplomatiche e dal nuovo assetto geopolitico dell'Europa ${ }^{7}$, che riscrisse la Carta del Vecchio Continente.

Le scelte di politica estera espresse dai geopolitologi italiani affondavano le loro radici nella tradizione risorgimentale dei padri della patria, come Giuseppe Mazzini, Carlo Cattaneo, Cesare Balbo e Vincenzo Gioberti, e nel colonialismo italiano, rafforzate dalla cosiddetta «Vittoria mutilata» promossa dalle grandi potenze europee (Francia e Gran Bretagna) ed extraeuropee (Stati Uniti) ai danni dell'Italia dopo la Grande Guerra. Lo scopo era quello di ridimensionare l'eventuale espansione territoriale italiana a causa della dissoluzione dell'impero austro-ungarico ${ }^{8}$. Una scelta che si rivelò esiziale $\mathrm{e}$ tale da esacerbare gli animi spingendo la gran parte dei popoli slavi ed europei - fra cui quello italiano - tra le braccia di un nazionalismo sempre più revanscista.

Le posizioni espresse dai geopolitologi in fatto di politica estera rappresentavano la continuazione di quella tradizione politico-geografica, rafforzata dall'unione a ridosso del primo conflitto mondiale fra nazionalismo e irredentismo, legata alle caratteristiche peculiari dei confini della nostra Penisola e dei suoi rapporti particolarmente stretti con tutte le terre irredente.

Giova ricordare che l'espansione coloniale ebbe origine in Italia a partire dal 1880, all'incirca un ventennio dopo il processo di unità nazionale. Il ritardo nella corsa alla spartizione delle colonie, rispetto alle principali potenze continentali, fu dovuto proprio alla tardiva unificazione. Il Mediterraneo era diventato infatti terreno di conquista dei

\footnotetext{
${ }^{6}$ La perdita di questi territori era avvenuta nel 1797 con il Trattato di Campoformio, che aveva cancellato dalla geografia politica dell'Europa la Repubblica di Venezia, primo degli Stati preunitari italiani a concludere la sua plurisecolare esistenza, e sancito il dominio austriaco su quelle terre un tempo controllate dalla Serenissima. Si veda al riguardo GHISALBERTI, Carlo, Adriatico e confine orientale dal Risorgimento alla Repubblica, Napoli, Edizioni Scientifiche Italiane, 2008, pp. 13-36. Al riguardo si veda anche GHISALBERTI, Carlo, Da Campoformio a Osimo. La frontiera orientale tra storia e storiografia, Napoli, Edizioni Scientifiche Italiane, 2001, pp. 15-17.

7 MASSI, Ernesto, «Geopolitica: dalla teoria originaria ai nuovi orientamenti», in Bollettino della Società Geografica Italiana, 1-6, 1986, pp. 3-45, pp. 8-11.

$8 \mathrm{Al}$ riguardo si veda MILANO, Rosario, MONZALI, Luciano, art. cit., pp. 103-127.
} 
francesi e degli inglesi, rafforzando oltremodo il loro interesse dopo l'apertura del Canale di Suez avvenuta nel 1869. A cominciare erano stati questi ultimi, con la pace di Utrecht del 1713 che sanciva il loro dominio su Gibilterra, porta di ingresso del Mare nostrum. Aveva in seguito interessato i francesi che nel 1842 avevano occupato Algeri per impossessarsi negli anni successivi del resto del Paese, mentre nel 1881 era stata la volta di Tunisi. Nel contempo, gli inglesi avevano proseguito nella loro penetrazione in direzione del Mediterraneo e avevano annesso al loro dominio prima Malta nel 1814, poi Cipro nel 1878 e, infine, l'Egitto nel 1882.

Un ritardo coloniale quello italiano provocato dalla divisione territoriale della Penisola in piccoli Stati, tanto che, mentre nel 1849 iniziava l'epopea coloniale del missionario britannico David Livingstone, a Roma falliva la difesa della Repubblica Romana guidata da Mazzini, Saffi e Armellini e capitolava Venezia, stremata dall'assedio austriaco. È quindi evidente l'enorme divario storico che separa il Risorgimento dalla scoperta dell'Africada parte del colonialismo europeo.

È stato tuttavia osservato 9 che il Risorgimento, seppur legato a un'epoca temporalmente e spiritualmente diversa, avrebbe comunque trasmesso alcune idee di base al colonialismo italiano, ovvero la missione del neonato regno, l'eredità imperiale romana da assumere, l'esaltazione dell'eroe e dei miti nazionali, in linea con le idealità di Mazzini, che chiedeva il controllo delle terre irredente e dell'Africa nord-orientale.

Ma andiamo per gradi e torniamo alle analisi compiute dai geografi triestini che favorirono la nascita della geopolitica.

L'interesse di Roletto per la geografia politica era stato delineato nel suo discorso di apertura dell'Anno accademico 1928-1929, dedicato a «La Geografia utilitaria», tenuto presso l'Ateneo triestino, in cui riprendeva un tema caro alla tradizione geografica italiana a partire dagli ultimi tre decenni dell'Ottocento fino ai primordi del Novecento, poi sviluppatosi ulteriormente con la Prima guerra mondiale e nel dopoguerra, ovvero quello di realizzare pienamente la diffusione di una Geografia politica ed economica al servizio dello sviluppo imperiale e del benessere della Penisola, che avesse i suoi cultori nelle aule universitarie e al contempo venisse divulgata a livello popolare allo scopo di rendere edotti della realtà geografica della nazione e delle sue potenzialità nel campo della politica internazionale i più ampi strati della popolazione, come già avveniva da decenni in altre potenze europee ed extraeuropee - soprattutto Francia ${ }^{10}$, Germania ${ }^{11}$,

\footnotetext{
9 GENTILE, Emilio, La Grande Italia. Il mito della nazione nel XX secolo, Roma-Bari, Laterza, 2006; CHABOD, Federico, Storia della politica estera italiana, 2 voll., Roma-Bari, Laterza, 1969; SALVATORELLI, Luigi, Pensiero e Azione del Risorgimento, Torino, Einaudi, 1963.

${ }^{10}$ Sulla Francia si veda il volume di SANGUIN, André-Louis, Vidal De La Blache. Un génie de la Géographie, Paris, Éditions Belin, 1993, in cui sono contenute alcune utili considerazioni sullo
} 
Gran Bretagna ${ }^{12}$ e Stati Uniti ${ }^{13}$ - e come si venne a delineare con l'avvento al potere di Benito Mussolini nelle scelte di politica estera dell'Italia, con la riforma scolastica di Giovanni Gentile e con l'istituzione della cattedra di Geografia politica ed economica nella facoltà di Scienze politiche nei principali Atenei della Penisola ${ }^{14}$, favorendo nel volgere di pochi anni la nascita dell'Istituto per gli Studi di Politica Internazionale (ISPI), che mantenne rapporti stretti con alcuni collaboratori del periodico «Geopolitica» e con lo stesso Roletto ${ }^{15}$.

Come accennato precedentemente, nel corso della sua prolusione triestina, Roletto tracciò le linee guida di quella che sarebbe stata la geografia politica italiana individuando i suoi precursori, soprattutto l'eredità lasciata da Cesare Correnti e dai padri della patria, per enfatizzare il ruolo e la funzione della geografia al servizio degli obiettivi della politica estera dell'Italia fascista. La coscienza geografica, dichiarò Roletto «è il lievito insostituibile dell'azione e dell'espansione, fondamento dell'educazione patriottica, sintomo dello sviluppo di una coscienza politico-

studio della geografia nel mondo francese dopo la sconfitta di Sedan (1870) e la Comune di Parigi (1871).

11 Per la Germania si veda CHIANTERA-STUTTE, Patricia, Il pensiero geopolitico. Spazio, potere e imperialismo tra Otto e Novecento, Roma, Carocci, 2015.

${ }_{12}$ Per quanto riguarda il mondo anglo-americano si può consultare con profitto il volume di PAGNINI, Maria Paola, SANGUIN, André-Louis, Storia e teoria della Geografia politica. Una prospettiva internazionale, Roma, Edicusano, 2014. Per la sola Gran Bretagna desta ancora un certo interesse il volume di MACKENZIE, John M., Propaganda and Empire. The Manipulation of British Public Opinion 1880-196o, Manchester, Manchester University Press, 1986.

13 Sugli Stati Uniti si veda PAGNINI, Maria Paola, Il contributo americano al pensiero geopolitico contemporaneo, in LIZZA, Gianfranco (a cura di), Geopolitica delle prossime sfide, Novara, De Agostini, 2011, in particolare pp. 295-320. Elementi utili sono contenuti anche nel volume di STEPHANSON, Anders, Destino Manifesto. L'espansionismo americano e l'Impero del Bene, Milano, Feltrinelli, 2004.

14 Sulle origini della facoltà di Scienze politiche nei vari Atenei italiani si vedano le seguenti pubblicazioni SIMONE, Gioia, Fascismo in cattedra. La Facoltà di scienze politiche di Padova dalle origini alla Liberazione (1924-1945), Padova, Padova University Press, 2015; COMPARATO, Vittor Ivo, LUPI, Regina, MONTANARI, Giorgio (a cura di), Le scienze politiche. Modelli contemporanei, Milano, Franco Angeli, 2011; LANCHESTER, Fulco (a cura di), Passato e presente delle Facoltà di Scienze politiche, Milano, Giuffrè 2003.

${ }_{15}$ DECLEVA, Enrico, Politica estera, storia, propaganda: L'ISPI e la Francia (1934-1943), ripubblicato in DECLEVA, Enrico, L'incerto alleato. Ricerche sugli orientamenti alleati dell'Italia unita, Milano, Franco Angeli, 1987, pp. 221-280; LOVISETTI, Giovanni, «I 60 anni dell'ISPI uno sguardo alle origini», in Relazioni Internazionali. Rivista trimestrale dell'Istituto per gli Studi di Politica Internazionale, LVII, 4/1993, pp. 87-96; MONTENEGRO, Angelo, Politica estera e organizzazione del consenso: note sull'Istituto di Politica Internazionale 19331943, in «Studi Storici» 19, 4/1978, pp. 777-817; GALIMI, Valeria, «"Un Ente che la scienza sposa alla propaganda". L'Istituto per gli studi di politica internazionale negli anni Trenta», in $I$ sentieri della ricerca. Rivista di Storia contemporanea, 6, 2/2007, pp. 147-163; GIONA, Francesco, L'ISPI, primo think-tank di politica internazionale, Roma, Aracne Editrice, 2014; VIGEZZI, Brunello, L'ISPI a Palazzo Clerici, in Palazzo Clerici. La proiezione internazionale di Milano, Milano, ISPI, 2004, pp. 199-207. 
nazionale» ${ }^{16}$. Fu lo stesso Roletto, al termine del suo discorso, a ricordare come questo stava avvenendo grazie alle nuove scelte di geografia politica a livello nazionale ed internazionale operate dal regime. Le posizioni espresse dallo studioso nel corso della prolusione risalivano alla moderna tradizione geografica italiana, fondata da Giuseppe Dalla Vedova nel 1880 $^{17}$ e sviluppata ulteriormente dallo stesso in un articolo del 1918, che faceva il punto sull'assioma espresso dal termine «Geografia utilitaria» ${ }^{18}$, impiegato più volte dal decano dei geografi italiani e tale da rappresentare la sintesi del binomio Geografia politica ed economica. Inoltre, nel 1931, lo stesso Roletto tornò sull'argomento, questa volta utilizzando lo pseudonimo di Giorgio La Corte, quando pubblicò sul periodico «La Coltura geografica. Rivista mensile di propaganda», da lui diretta con l'apporto di Massi quale capo-redattore, un contributo sulla figura di Dalla Vedova $^{19}$, in cui l'autore ricordava il sostegno espresso dal decano dei geografi italiani in favore di una scienza del territorio che fosse al servizio della nazione e finalizzata al suo benessere. «E anche l'ultimo suo scritto, La Geografia nella scuola e nella vita moderna (Nuova Antologia, 1918), - ricordò Roletto nel suo articolo - dettato sul volgere dell'ottantacinquesimo anno improntato ad una mirabile lucidità di forma ed equilibrio di pensiero, è una battaglia: idee e fatti vi si addensano con tale efficacia suggestiva che vano sarebbe negare o ridurre il valore educativo della Geografia nella scuola, che è la fucina dell'avvenire della patria».

Nel quadro delle analisi compiute dagli studiosi sulla rivista «Geopolitica», il Mediterraneo venne così a rappresentare il riferimento imprescindibile, per la evidente collocazione geografica dell'Italia, in linea con quanto era avvenuto nell'antichità con il sorgere e l'espandersi dell'Urbs eterna ${ }^{20}$, che aveva conseguito il dominio del Mare nostrum e di tutto l'ecumene conosciuto all'epoca, in virtù dell'impegno militare delle

16 ROLETTO, Giorgio, La geografia come scienza utilitaria, «Discorso inaugurale dell'a.a. 19281929», Regia Università degli Studi economici e commerciali di Trieste, 1929, pp. 3-20.

17 Il termine «utilitarismo» era messo in stretto rapporto con quella che nella seconda metà dell'Ottocento veniva definita la Geografia economica e commerciale. Esso serviva ad indicare ciò che è «utile», non nel senso di più facile e conveniente, ma che è finalizzato al meglio, ovvero al benessere individuale e sociale, ovvero del singolo e della società. Solo più tardi, a partire dal 1918, la «Geografia utilitaria» verrà associato dallo stesso Dalla Vedova alla Geografia politica ed economica. Il vocabolo «utilitarismo» venne menzionato dal geografo per la prima volta nel contributo di DALLA VEDOVA, Giuseppe, «La Geografia a' giorni nostri», in «Nuova Antologia», maggio 1873, ristampato in DALLA VEDOVA, Giuseppe, Scritti geografici (18631913). Scelti, coordinati e ripubblicati a cura d'un Comitato di geografi in occasione dell' $80^{\circ}$ genetliaco dell'Autore, Novara-Roma, Istituto Geografico De Agostini, 1914, pp. 15-69, p. 19.

18 DALLA VEDOVA, Giuseppe, «La Geografia nella vita e nella scuola moderna», in Nuova Antologia, CXCVI, 1918, pp. 223-233.

19 LA CORTE, Giorgio, «Giuseppe Dalla Vedova», in La Coltura geografica. Rivista mensile di propaganda, II, 9/1931, pp. 224-226, p. 226.

${ }^{20}$ MORICHINI, Ugo, "Geopolitica romana. La fase propedeutica», in Geopolitica, I, 3/1939, pp. 153-159. 
sue legioni. I presupposti per la nascita della geopolitica italianarisalivano ancheall'attività rivoluzionaria e alle idee politiche enunciate nei suoi scritti da Giuseppe Mazzini, che sempre invocò il ruolo civilizzatore e la missione dell'Italia nel Mediterraneo logica evoluzionedella Terza Roma, a cui si aggiunse la visione dottrinaria espressa dagli altri padri della patria, favorevoli al pieno controllo delle terre irredente e dei territori africani prospicienti alla Penisola, dove vivevano numerosi compatrioti ${ }^{21}$.

Fusoprattutto,però,a partiredalla presidenza del Consiglio affidata a Francesco Crispi e connotata da un deciso nazionalismo, che si tentò di impedire il progressivo e assoluto controllo del Mediterraneo e dell'Africa nord-orientale da parte della Francia e della Gran Bretagna. Dal canto loro, i successori di Crispi, ovvero i governi guidati dai liberali fino al primo dopoguerra, si orientarono verso una politica estera che si ispirava ai problemi dell'Europa centrale e balcanica in linea con la tradizione realista liberale e all'interventismo nazionaldemocratico di Carlo Sforza. Tuttavia, durante la presidenza di Giovanni Giolitti venne deciso l'intervento per la conquista della Libia (1911-1912) a spese dell'impero ottomano in crisi profonda ${ }^{22}$. Al contempo, l'esercito italiano prese il controllo delle Sporadi meridionali, mettendo in guardia gli ottomani da qualsiasi intervento in Africa a sostegno della resistenza libica.

L'avvento al potere del Fascismo nell'ottobre 1922 destò agli inizi una certa preoccupazione da parte degli osservatori, che venne poi fugata dalle scelte ponderate del regime, il quale decise di evitare svolte radicali nella politica internazionale dell'Italia ${ }^{23}$.

Successivamente, la politica estera di Benito Mussolini si orientò a partire dagli anni Trenta sulla base della nuova realtà geopolitica mondiale, a dimissionare nel 1932 Dino Grandi da ministro degli Esteri e ad assumere la carica da parte dello stesso Mussolini. Il duce decise di rivolgere la politica estera del Fascismo in direzione del controllo del Mediterraneo, valutando un'oculata attività di dominio sul Canale di Gibilterra e quello di Suez. Il regime era infatti consapevole dell'importanza legata al controllo dei due stretti, soprattutto per la vita politico-commerciale presente e futura della nazione in vista di una rinnovata tendenza espansionistica dell'Italia. Nel 1938, il

${ }^{21}$ Si veda a riguardo LEVIS SULLAM, Simon, L'apostolo a brandelli. L'eredità di Mazzini tra Risorgimento e fascismo, Roma-Bari, Laterza, 2010. Alla stessa stregua anche BENEDETTI, Paolo, «Mazzini in "camicia nera" I», in Annali della Fondazione Ugo La Malfa, XXII, 2007, pp. 163-206; BENEDETTI, Paolo, «Mazzini in "camicia nera" II», in Annali della Fondazione Ugo La Malfa», XXIII, 2008, pp. 159-184.

${ }_{22}$ MICHELETTA, Luca, UNGARI, Andrea (a cura di), L'Italia e la guerra di Libia cent'anni dopo, Roma, Studium, 2013.

23 MILANO, Rosario, MONZALI, Luciano, art. cit., pp. 128-129. 
75 per cento delle importazioni italiane e il 98 per cento di quelle petrolifere raggiungeva la nostra Penisola grazie alla passaggio delle navi attraverso lo stretto di Gibilterra e di Suez. Nel 1940, il numero delle importazioni italiane di materie prime e derrate alimentari era salito all'8o per cento, laddove le principali potenze europee, avversarie dell'Asse, controllavano militarmente i due canali.

Il processo di gestazione della rivista si legò in particolare all'evoluzione stessa della dottrina geopolitica italiana richiedendo per questo alcuni anni prima che si giungesse alla sua completa realizzazione sul piano editoriale. Fu uno dei due condirettori, Massi, a ricordare, in un articolo del 1940, "L'ora della Geopolitica», pubblicato sul periodico «Critica fascista» dell'allora ministro dell'Educazione Nazionale Giuseppe Bottai, che le origini della geopolitica italiana risalivano alla metà del 1938, mentre prima si poteva parlare soltanto di geografia politica.

Tuttavia, precisò alcuni decenni dopo lo stesso Massi che, nonostante l'esistenza di una geopolitica tedesca nata nel 1924, anno di pubblicazione del primo fascicolo della rivista «Zeitschrift für Geopolitik» del geopolitologo Karl Haushofer, in realtà i presupposti epistemologici e storici della geopolitica italianacostituirono una risposta allo sviluppo di analoghe tendenze già in voga in Germania e in Francia e furono assolutamente di origine autoctona, legati perciò alla tradizione geografica italiana che affondava le sue radici nelle opere dei padri del Risorgimento, di cui sopravvivevano nella geografia moderna alcuni importanti postulati di statistica e di economia, congiunti a diversi assiomi epistemologici mutuati dalla tradizione filosofico-culturale di Giandomenico Romagnosi e dei suoi allievi Carlo Cattaneo e Melchiorre Gioia, prima della piena e completa sistematizzazione antropogeografica compiuta dal tedesco Friedrich Ratzel, alla fine dell'Ottocento ${ }^{24}$. L'evoluzione delle moderne scienze territoriali italiane, costituirono il frutto dell'attività scientifica compiuta a partire dalla seconda metà dell'Ottocento da Giuseppe Dalla Vedova ${ }^{25}$, grazie ai suoi studi compiuti a Vienna e ai suoi rapporti con i geografi tedeschi, nonché dalla scuola fiorentina di Giovanni e Olinto Marinelli.

Le scuole geografiche create da questi studiosi italiani con l'apporto dei loro allievi favorirono, tra il 1873 e la Prima guerra mondiale, fino all'avvento del Fascismo, la nascita e lo sviluppo della Geografia moderna in Italia, postulando assiomi

24 LANDO, Fabio, La Geografia di Friedrich Ratzel: suolo, stato e popolo, in Bollettino della Società Geografica Italiana, 5/2012, pp. 477-512.

25 LUZZANA CARACI, Ilaria, La Geografia italiana tra '80o e '9oo (dall'Unità a Olinto Marinelli), Genova, Università di Genova - Facoltà di Magistero, Pubblicazioni dell'Istituto di Scienze geografiche, 1982, pp. 11-30; LUZZANA CARACI, Ilaria, A sessant'anni dalla morte di Giuseppe Dalla Vedova, Genova, Università di Genova, Facoltà di Magistero, 1978, pp. 4-22. 
fondamentali per la genesi e l'evoluzione della Geografia politica ed economica italiana, poi Geopolitica, frutto della sintesi tra Geografia fisica e Geografia umana con una decisa prevalenza per quest'ultima nei suoi sviluppi di matrice antropogeografica ${ }^{26}$.

Dal canto loro, i nostri geopolitologi ritenevano che le scuole tedesca e francese fossero troppo chiuse nel loro dogmatismo: la prima perché sosteneva l'espansione della Germania, la seconda invece era troppo interessata a mantenere i confini mitteleuropei imposti con il Trattato di Versailles. I tedeschi fondavano la loro geopolitica sul «determinismo» geografico, mentre i francesi sul «possibilismo» 27 . Per Massi, invece, era necessario che entrambi concorressero alla nuova scienza, ma con l'aggiunta di un terzo elemento: «l'umanesimo geografico». Con quest'ultima espressione si intendeva valorizzare i fattori culturali e spirituali che appartenevano fortemente alla tradizione nazionale italiana, che muovevano da Dante e Petrarca fino a Macchiavelli e Giovanni Botero, dal punto di vista politico-culturale e letterario, ma soprattutto da Giambattista Vico, e che si perpetuavano nelle dottrine filosofiche e politico-economiche dei padri del Risorgimento: Romagnosi, Cesare Balbo, Vincenzo Gioberti, Mazzini, Cattaneo, Melchiorre Gioia, Carlo Pisacane.

«Essenza della geopolitica italiana fu l'antideterminismo - sottolineava Massi perché non poteva considerare l'uomo e le società umane incapaci di superare i fenomeni ambientali visti nella morsa ferrea di leggi statistiche» ${ }^{28}$. In sintesi, «la geopolitica italiana è la scienza degli spazi vitali, tesa all'optimum delle aree nelle quali ambiente, tradizioni storiche, necessità di vita presenti e future si concordano in vista del bene comune» 29 .

26 RINAURO, Sandro, La conoscenza del territorio nazionale, in CASSATA, Francesco, POGLIANO, Claudio (a cura di), Storia d'Italia. Annali 26. Scienze e Cultura dell'Italia unita, Torino, Einaudi, 2011, pp. 497- 523, pp. 507-508.

27 Una disamina puntuale sulle due scuole di geopolitica è contenuta nell'articolo di MASSI, Ernesto, «Nuovi indirizzi della geografia politica in Francia», in Rivista Internazionale di Scienze Sociali, 5, 2/1938, pp. 194-208.

${ }^{28}$ MASSI, Ernesto, «Il contributo della geopolitica alla comprensione del mondo moderno», in Partecipare. Rivista di studi corporativi, XXII, 2/1992, pp. 128-145, p. 136. Si veda anche ANTONSICH, Marco, La coscienza geografico imperiale del regime fascista, 'Geopolitica' (1939-1942), Tesi di laurea discussa presso l'Università Cattolica del Sacro Cuore di Milano, Facoltà di Scienze Politiche, a.a. 1990/1991, p. 9, in cui afferma: "Ciò può allora spiegare il perché la nostra geopolitica,in contrasto ad esempio con quella tedesca, improntata a concezioni più deterministiche, si sia sempre riconosciutain una frase del geografo genovese Goffredo Jaja: 'è lo spirito che doma la materia'. L'elemento "volontà", ovvero la possibilità che in fondo l'Uomo possa in ogni momento mutare il cammino impresso alle cose della Natura può considerarsi come il presupposto comune degli articoli dei nostri geopolitici, i quali, infatti, sempre consideraronole proprie leggi come 'tendenziali' e mai come 'assolute'. Una posizione, insomma, diversa ancora da quelladella geopolitica francese, legata all'umanesimo-possibilista di Vidal de la Blache».

29 MASSI, Ernesto, «Il contributo della geopolitica alla comprensione del mondo moderno», cit., p. 136. 
Uno degli argomenti principali delle analisi compiute sul periodico dagli studiosi delle diverse discipline - come abbiamo osservato in precedenza - divenne il Mediterraneo e il pieno dominio del suo bacino da Oriente a Occidente, in direzione dell'Africa, dei Balcani, del Vicino Oriente e delle isole delle Sporadi meridionali, da parte della nostra Penisola, sull'onda delle scelte espansionistiche ed imperiali decise dal regime fascista, giustificate da parte dei fondatori e dei collaborati di «Geopolitica» sulla base della collocazione geografica dell'Italia, situata al centro del Mediterraneo, e della tradizione risorgimentale mutuata dal pensiero di Giuseppe Mazzini30, fautore della Terza Roma. L’Urbe eterna venne a costituire un punto di riferimento costante per gli studiosi di «Geopolitica» e tale da rappresentare il simbolo delle strategie di espansione imperiale raggiunte progressivamente nel corso dei secoli, che fecero del bacino del Mediterraneo un'area di competenza esclusiva dell'antica Roma, grazie all'impegno costante delle sue legioni.

Sul primo numero della rivista pubblicato nel gennaio 1939, Roletto e Massi, in un contributo teso a sottolineare le istanze e gli obiettivi della geopolitica italiana ${ }^{31}$, affermarono che nell'ambito dei nuovi rapporti instaurati dal fascismo tra politica e scienza, la geografia italiana aveva nuovi compiti da assolvere. Il ruolo che le scienze territoriali della nostra Penisola sarebbe stato quello di comprendere le complessità crescenti e le interconnessioni tra gli eventi a livello mondiale. In questo modo, era più che mai evidente che la geografia aveva ereditato un ampio settore completamente nuovo di studio e di analisi, che le imponeva maggiori responsabilità rispetto al passato. In quel momento storico alle scienze geografiche si rivolgevano le altre discipline per comprendere e spiegare la realtà mutevole del mondo moderno, grazie alle capacità stesse della geografia di riassumere e analizzare il ruolo svolto dai singoli eventi a livello mondiale.

Nell'articolo le critiche dei due geopolitologi erano rivolte soprattutto alle posizioni espresse dagli studiosi di geopolitica tedeschi e francesi. Gli uni, colpevoli di essere legati ad una visione troppo determinista, fondata sul ruolo preponderante della natura sull'uomo. Gli altri, invece, troppo indotti a difendere esclusivamente gli interessi nazionali per mantenere invariatolo status quo impostocon la pace di Versailles e i trattati successivi, da parte della Francia e della Gran Bretagna, alle altre nazioni europee.

30 Per Mazzini, l'Italia avrebbe dovuto acquisire il dominio su alcune aree dell'Adriatico, dei Balcani e in particolare dell'Africa settentrionale: Tunisia, Algeria e Libia. SCOCCHI, Angelo, «L'Italia e i Balcani nel pensiero di Giuseppe Mazzini», in Geopolitica, III, 11/1941, pp. 486-490. ${ }^{31}$ ROLETTO, Giorgio, MASSI, Ernesto, «Per una geopolitica italiana», in Geopolitica, I, 1/1939, pp. 5-11. 
Giova ricordare, infatti, che le scelte dei direttori e dei collaboratori del periodico scaturirono per ottenere il loro sostegno alle nuove direttive stabilite dal regime fascista e per garantire, al contempo, una palingenesi dell'Italia nel contesto della politica interna ed internazionale. I geopolitologi si ripromettevano di annullare gli esiti della Prima guerra mondiale, con la cosiddetta "Vittoria mutilata", affinché non fosserovissuti passivamente come all'epoca dei governi liberali, ma rappresentassero delle rinnovate possibilità legate agli interessi della politica italiana, garantendo alla nazione l'occasione di gareggiare senza esitazioni e tentennamenti nel grande agone mondiale. La loro speranza era soprattutto quella di poter determinare o quanto meno influenzare positivamente le scelte di politica estera del regime.

Per questo, è utile ricordare che la rivista ospitò analisi su tutti i temi che riguardavano la geopolitica a livello mondiale con l'apporto di docenti afferenti alle diverse discipline universitarie, focalizzando sempre più l'attenzione su argomenti legati al conflitto, ma non disdegnando di contemplare lo studio teoretico e dottrinario della geopolitica, insieme ad argomenti che riguardavano la storia della geografia italiana e della stessa Penisola.

Lo stesso Mussolini non aveva fatto mistero delle scelte future nell'ambito della politica internazionale, osservando che il fattore geografico imponeva le sue direttive ad una tra le maggiori attività dello Stato, ovvero la politica estera, affermando il 15 dicembre 1924, durante un intervento al Parlamento italiano, che «la politica estera è la proiezione globale e complessa di una nazione nel mondo»32. Questa attività legata alla vita della nazione veniva considerata da Mussolini, frutto di determinate condizione geografiche, storiche ed economiche: «Una politica non è mai originale. La politica estera è strettamente condizionata a circostanza di fatto nell'ordine geografico, nell'ordine storico e nell'ordine economico»33.

Un decennio più tardi, il 18 marzo 1934, Mussolini fu ancora più esplicito affermando, alla seconda Assemblea quinquennale del regime, che «la geografia è il dato immutabile che condiziona i destini ai popoli»34. Espressione che verrà riportata qualche anno dopo anche dai geopolitologi triestini sulla rivista creata per sostenere e indicare le linee guida della politica imperiale del regime fascista.

Del resto, non erano nuove le posizioni assunte dagli studiosi delle scienze territoriali come quelle di Antonio Renato Toniolo, legate alla tradizione risorgimentale che difendeva la piena realizzazione dell'unità nazionale, nonostante i parziali successi

$3^{2}$ MUSSOLINI, Benito, Scritti e discorsi, Roma, Istituto Poligrafico dello Stato, 1939, vol. IV, p. 381 .

33 ID., op. cit., Roma, Istituto Poligrafico dello Stato, 1939, vol. IV, p. 382.

34 ID., op. cit.,Roma, Istituto Poligrafico dello Stato, 1939, vol. IX, p. 39. 
ottenuti con la Grande Guerra, e in linea con le scelte di politica estera del Fascismo e dei collaboratori della Scuola di Mistica fascista35, riguardo alle terre irredente che venivano considerate come parte integrante della Penisola italiana e come strumento di dominio del Mediterraneo, ovvero la Corsica, la Dalmazia ${ }^{36}$, Nizza, la Savoia ${ }^{37}$ e l'isola di Malta. Analogamente, un altro collaboratore di «Geopolitica» Livio Chersi sottolineava che il Mediterraneo dall'antica Roma in poi, salvo il periodo dell'espansionismo islamico, è sempre stato un mare sotto il controllo dell'Italia ${ }^{3}$. Per questo era necessario che gli interessi vitali dell'Italia fossero difesi allo scopo di contrastare, se non annullare, il dominio economico-militare britannico che controllava le vie di accesso agli Oceani, dallo stretto di Gibilterra a quello di Suez. Non si poteva evitare perciò che, dal momento in cui era stato proclamato l'impero da parte di Mussolini, l'Italia doveva diventare uno Stato con interessi a livello mediterraneo ed europeo. Chersi non mancava di ricordare che le condizioni necessarie ad una pace e allo sviluppo del bacino mediterraneo e della stessa Europa erano strettamente legate alla libertà e all'effettiva sicurezza dell'Italia in seno al Mediterraneo e ai due canali di accesso al Mare nostrum. A sua volta, nel 1942, un altro studioso, Lodovico Magugliani, pubblicò un contributo apparso nel numero doppio della rivista dell'agosto-settembre dello stesso anno39, che analizzava la funzione storica del Mediterraneo, considerato come il mare che nel corso delle varie epoche era stato in grado di determinare i movimenti ascensionali delle civiltà e che era capace di riorganizzare il ritmo dei periodi di regresso oppure quelli di stasi. Per questo, osservava ancora Magugliani, il Mediterraneo costituì sempre un nodo delle correnti afferenti ed efferenti dei traffici, di tutte le necessità geografiche, economiche e vitali dell'ecumene. Qualche mese dopo, siamo nel novembre 1942, Magugliani sempre dalle pagine del periodico, pubblicò l'altro articolo, in cui veniva analizzato il rapporto fra il Mare nostrum, l'Africa e il continente eurasiatico. In tale contesto l'Italia veniva a

35 CAMUSSI, Renato, CARATI, Angelo, CARELLI, Gustavo, BELLOTTI, Romeo, CALEFFA, Giorgio (a cura di), Diritti italiani nel mondo: Corsica, Nizza e Savoia, Tunisi, Suez e Gibuti, Prima edizione dei Quaderni della «Scuola di Mistica Fascista», editi a cura della rivista «Dottrina Fascista», 1938.

${ }^{36}$ TONIOLO, Antonio Renato, «Politica e Geografia», in Giornale di Politica e Letteratura, 6, 4/1930, pp. $1-24$.

37 TONIOLO, Antonio Renato, «Ragioni geografiche dell'italianità della Corsica», in Bollettino della R. Società Geografica Italiana, V, 7-8/1940, pp. 435-444.

${ }^{38}$ CHERSI, Livio, «Problemi geopolitici del Mediterraneo», in Geopolitica, II, 5/1940, pp. 214215, p. 214.

39 MAGUGLIANI, Lodovico, Impostazione geopolitica del bacino Mediterraneo,in «Geopolitica», IV, 8-9/1942, pp. 374-382. 
rappresentare un ponte di collegamento tra le due realtà continentali ${ }^{\circ}$ e il centro di un nuovo blocco geopolitico.

Giova ricordare che alcuni tra i collaboratori di «Geopolitica», come Luigi Filippo De Magistris, docente di Geografia politica ed economica all’Università Bocconi di Milano, e molti altri studiosi tra storici e geografi sostennero apertamente la politica estera fascista e le sue strategie di dominiolungo tutto il Mediterraneo e nei territori limitrofi, giustificando la scelta come un dato inoppugnabile di natura geopolitica.

Durante le lezioni tenute dagli anni Venti agli anni Quaranta per il corso di Geografia politica ed economica, presso l'Università Bocconi di Milano, De Magistris sottolineò lo stretto rapporto tra Italia e Mediterraneo, soprattutto in ragione dei suoi confini, per la sua conformazione geografica e per la presenza dei nostri connazionali, pari al 50 per cento della popolazione complessiva, in tutte le terre limitrofe al Mare nostrum. Una superiorità demografica che, a detta del De Magistris, motivava il dominio dell'Italia sul bacino del Mediterraneo e sui territori prospicienti.

Del resto, era stato lo stesso Mussolini a ribadire l'importanza del controllo sul Mare nostrum, quando aveva sottolineato, in un discorso tenuto a Milano il 1 novembre 1936, che il Mediterraneo, considerato dall'Inghilterra una mera arteria di comunicazione, aveva invece per l'Italia il valore di uno spazio vitale, al quale non potevamo assolutamente rinunciare ${ }^{41}$.

Nel 1940, dalle pagine di «Geopolitica» era intervenuto Massi per affermare che «se il riassetto mondiale dovrà basarsi sull'organizzazione di grandi spazi terrestri, all'Italia spetterà il compito di presiedere all'organizzazione dello spazio mediterraneo» ${ }^{42}$. Tuttavia, proseguendo nella sua disamina il geografo triestino aveva fatto notare che nel bacino non vi era né uniformità né omogeneità, al contrario si poteva osservare la massima eterogeneità di forme naturali e manifestazioni di vita, «forme e manifestazioni che si presentano però così strettamente associate da comporsi in unità, quando le condizioni storiche ne favoriscono il coordinamento e l'organizzazione per virtù di forze politiche»43. Ma questa unità - chiosò il geografo - andava costruita e conquistata dall'impero italiano. Soltanto l'Italia - annotava Massi - perseguiva tenacemente, dall'avvento del Fascismo, una politica di costruzione e di espansione

$4^{40}$ MAGUGLIANI, Lodovico, «Il Mediterraneo centro geopolitico del blocco continentale EuroAsio-Africano», in Geopolitica, IV, 11/1942, pp. 495-504.

${ }^{41}$ ATKINSON, David, Geopolitics and the geographical imagination in Fascist Italy. A Doctoral Thesis. Submitted in partial fulfillment of the requirements for the award of Doctor of Philosophyof the Loughborough University of Technology, Loughborough, a.a. 1994-1995, p. 222.

${ }^{22}$ MASSI, Ernesto, Problemi mediterranei, in «Geopolitica», II, 12/1940, pp. 331-340, p. 331.

43 Ibidem. 
mediterranea, che a distanza di tempo avrebbe portato i suoi frutti: forte della sua posizione, delle virtù del suo popolo, della ricchezza delle sue tradizioni, dell'universalità dei valori che rappresentava nel mondo, era conscia della sua missione «in questo mare che è il suo mare, in questa regione che è il suo spazio di vita. Non a torto perciò, studiosi, pensatori illustri e scrittori, hanno portato la loro attenzione ai problemi mediterranei che l'Italia dovrà affrontare nella sua opera ricostruttrice e riordinatrice» 44 .

Per realizzare quanto affermato, sottolineò Massi, era necessario annullare la presenza inglese dal Mediterraneo, azione questa che avrebbe costituito il presupposto per ogni realizzazione unitaria, affinché Grecia e Turchia tornassero a presiedere la «Porta Orientale» in funzione mediterranea «essendo restituite alla loro naturale missione geopolitica. I popoli arabi potranno evolversi naturalmente e afferrare la mano offerta da Roma. L'Egitto potrà liberamente svilupparsi, nella valle del fiume che è la sua vita. Altre situazioni nuove già s'intravvedono. La Francia, diminuita territorialmente a settentrione e non più legata oltre Manica, accentuerà la sua gravitazione mediterranea. L'Italia e la Spagna chiamate più largamente a colonizzare il Nord-africa, rafforzeranno i legami eurafricani tra le opposte sponde. Le porte del Mediterraneo, aperte al transito dei mediterranei e liberate da esosi controlli stranieri, saranno origine di una prima solidarietà politica tra popoli mediterranei, basata sul comune interesse a mantenerle libere e garantirne ladifesa. La funzione che potrà esercitare l'Italia a tale riguardo risulta evidente»45. Tuttavia, il progetto di riassetto del Mediterraneo nel quadro del suo riordino geostrategico e geo-economico era in realtà più ambizioso ed organico nelle sue linee evolutive in rapporto con la nostra Penisola. Per questo, Massi osservò che, «altra ragione di solidarietà politica sarà lo sviluppo dei grandi spazi contigui al Mediterraneo, specialmente dello spazio vitale tedesco e della regione russa, sviluppo che suggerirà agli Stati mediterranei di polarizzarsi per ragioni di equilibrio intorno all'Italia» ${ }^{6}$.

Del resto la nuova visione del regime fondata sul riassetto territoriale e marittimo del Mediterraneo non era nuova per la geopolitica italiana. Infatti, la questione riguardante il pieno dominio sul Mare nostrum aveva interessato anche altri geografi, come Toniolo quando, durante una conferenza tenuta all'Accademia degli Agiati di Rovereto, il 15 maggio 1940, pubblicata successivamente sul periodico «Geopolitica»47,

\footnotetext{
44 Ibidem.

45 Ibidem, p. 334.

46 Ibidem.

47 TONIOLO, Antonio Renato, L'unità economica e politica del Mediterraneo, in «Geopolitica», II, 12/1940, pp. 165-169.
} 
aveva ricordato la realtà storica del dominio imperiale di Roma sul Mediterraneo e la sua importanza, assimilabile a quella che era stata raggiunta nel mondo antico con la Roma dei Cesari. Il docente dell'Università di Bologna aveva osservato che l'Italia e la Spagna avrebbero dovuto cooperare attivamente alla realizzazione da parte di entrambe del pieno controllo di tutta l'area del Mediterraneo, affinché le due nazioni potessero annullare definitivamente l'ingombrante presenza inglese in seno al suddetto bacino.

Nel 1940, Renata Pess, studiosa afferente alla scuola triestina di Roletto,aveva analizzato sulle pagine del periodico il ruolo fondamentale svoltodall'Albania dal punto di vista geopolitico ${ }^{48}$. La geografa aveva ricordato che l'Italia era un Paese mediterraneo e l'Albania rappresentava una dellemaggiori realtà della vita del Mediterraneo. La scelta del pieno controllo dell'Albania era importante per tre motivi: geografico, storico e politico. Dal punto di vista geografico, precisava la Pess, l'Albania dista soltanto 75 $\mathrm{km}$. dalle coste pugliesi, per cui si poteva considerare questa nazione come un'estensione naturale della stessa costa italiana. A detta della studiosa, la realtà del territorio e delle sue coste aveva orientato l'Albania in direzione dell'Adriatico49.

Inoltre, precisava la geopolitologa, che la realtà idrologica di questa nazione dei Balcani è diretta verso Occidente, in direzione dell'Italia, tanto che storicamente e culturalmente le influenze della nostra Penisola su Tirana erano indubbiamente elevate. Per questo era necessario, che non fosse più uno Stato indipendente, ma che alla nazione albanese fosse garantita una difesa amichevole e una guida disinteressata da parte dell'Italia. Il braccio di mare che separa l'Italia dallo Stato dei Balcani occidentali, attraverso lo Stretto di Otranto, sottolineava la Pess, ha permesso al popolo albanese di svilupparsi separatamente rispetto alle altre razze balcaniche. Inoltre, l'Albania svolgeva un ruolo molto importante dal punto di vista politico per l’imperialismo italiano, poiché con la conquista dell'impero africano, il bacino orientale del Mediterraneo veniva a costituire il fulcro dell'impero italiano. La conseguenza naturale e logica della visione imperiale dell'Italia - affermava la geografa - era quella di portare i popoli balcanici a vivere in pace e nel benessere economico. Inoltre, il pieno

\footnotetext{
48 PESS, Renata, «Aspetti geopolitici dell'Albania», in Geopolitica, II, 1/1940, pp. 9-16.

49 BECHERELLI, Alberto, CARTENY, Andrea (a cura di), L'Albania indipendente e le relazioni italo-albanesi (1912-2012). Atti del Convegno in occasione del Centenario dell'Indipendenza albanese - Sapienza, 22 novembre 2012, Roma, Edizioni Nuova Cultura, 2013; MICHELETTA, Luca, Il sostegno alla Grande Albania: il caso Kosovo, in CACCAMO, Francesco, MONZALI, Luciano (a cura di), op. cit., pp. 257-308; PAÇUKAJ, Sokol, Albania. Antropografia degli anni '2o, Roma, Edizioni Nuova Cultura, 2012; RAGO, Paolo, Tradizione, nazionalismo e comunismo nell'Albania contemporanea, Roma, Edizioni Nuova Cultura, 2011; BIAGINI, Antonello, Storia dell'Albania contemporanea, Milano, Bompiani, 2005; MINUNCO, Giuseppe, Albania nella storia, Lecce, Besa, 1995; ROSELLI, Alessandro, Italia e Albania: relazioni finanziarie nel ventennio fascista, Bologna, Il Mulino, 1986.
} 
dominio sull'Albania avrebbe garantito un aumento delle esportazioni italiane verso la Grecia, la Jugoslavia, e presumibilmente anche in direzione della Bulgaria e della Romania. Il controllo dell'Albania da parte dell'Italia avrebbe permesso di rafforzare il dominio sul Mediterraneo orientale, circumnavigando la presenza marittima britannica nel bacino del Mare nostrum.

Altrettanto importante era nella visione geopolitica dei geografi italiani il ruolo svolto dalla Bulgaria, che forte della presenza dell'Italia in Albania, della Germania sul Danubio e dell'Ungheria aumentava la potenza dello schieramento balcanico dell'Asse, perciò «tra le incertezze e le esitazioni della penisola balcanica - affermava l'autore anonimo dell'articolo -, il triangolo Albania-Bulgaria-Ungheria costituisce una realtà operante, positiva, precisa» ${ }^{5}$. La normalizzazione dei rapporti con la Jugoslavia ha rafforzato la posizione della Bulgaria nel confine occidentale, l'ingrandimento dell'Ungheria ha attenuato la pressione romena, l'unione dell'Albania alla nostra Penisola ha rallentato la pressione greca. Le rivendicazioni bulgare, mai abbandonate, avrebbero acquisito un maggior rilievo ed erano diventate un fattore determinante della politica balcanica, essendo le direttrici geopolitiche naturali dell'espansione di Sofia: ad oriente lungo il Danubio, verso la foce del fiume; a sud lungo la Mariza, verso l'Egeo. Direttrici che attraversano terre un tempo parte integrante della Bulgaria: la Dobrugia, ceduta alla Romania dopo l'ultima guerra balcanica, la Tracia, perduta in parte nel 1913 e in parte nel 1918, divisa tra Grecia e Turchia dopo la guerra di rivincita dal leader turco Kemal Atatürk. Decisamente forte era inoltre l'esigenza vitale di Sofia per uno sbocco al mare in direzione dell'Egeo.

Ad esplicitare e giustificare la strategia del Fascismo nei confronti del Mediterraneo fu, sempre nel 1939, Renzo Sertoli Salis ${ }^{51}$, docente di Diritto coloniale e di Diritto internazionale, che ricordò come nel pensiero del duce la posizione geografica dell'Italia al centro del Mare nostrum si era sempre estrinsecata in una politica di cordiali rapporti con tutti i Paesi rivieraschi, dalla Spagna alla Grecia e dalla Turchia all'Egitto, per mirare alla formazione nel Mediterraneo di un blocco a sé per quanto possibile indipendente dalle cosiddette talassocrazie occidentali. L'occupazione italiana dell'Albania aveva suscitato emozioni in Jugoslavia e Grecia, così come era avvenuto in Turchia, annunziando una dichiarazione di mutua assistenza con la Gran Bretagna, il 12 maggio 1939. Tuttavia, precisò Sertoli Salis, di lì a poco si comprese che l'Albania, come era avvenuto del resto in passato con le isole italiane dell'Egeo, rappresentava un

50 S.a., «Balcania Meridionale», in Geopolitica, I, 7-8/1939, pp. 395-397, pp. 396-397.

51 SERTOLI SALIS, Renzo, «La guerra europea, il Mediterraneo orientale e l'Italia», in Geopolitica, I, 10/1939, pp. 522-526. 
punto di forza ma non un trampolino di lancio per ulteriori conquiste. Per cui queste considerazioni avevano riaperto la strada al riavvicinamento della Grecia e della Turchia all'Italia. Tuttavia la politica portata avanti dal leader Atatürk, in momenti di difficoltà si era rivolta alla Russia sovietica, a cui era legata anche per ragioni commerciali e qualora si fossero verificati i pericoli di un conflitto con Mosca, le tensioni non avrebbero fatto altro che riavvicinare Ankara alla Gran Bretagna.

Nel 1941, fu la volta di Gustavo Carelli52, allievo di Massi, con cui si era laureato in Geografia politica ed economica, presso la facoltà di Scienze politiche dell'Università di Pavia. Carelli analizzò un tema particolarmente caro all'Italia: la Dalmazia. Da poco conquistata dall'esercito italiano, legata dal punto di vista politico alla Jugoslavia, ma grazie all'altipiano dinarico separata dal nuovo Stato, nato dopo la pace di Versailles, econnessa, invece, alla tradizione culturale della nostra Penisola, così come dal punto di vista climatico, geografico, antropico ed economico, soprattutto per la presenza secolare dei nostri connazionali.

La storia, precisò Carelli, giustificava la presenza dell'Italia e dei suoi cittadini nella regione dei Balcani occidentali, tanto che Roma e Venezia avevano garantito in quell'enclave il mantenimento e la difesa della civiltà latina impedendo l'infiltrazione di etnie straniere, come quella slava. Più tardi, il ruolo della Dalmazia era stato ancora una volta lo stesso, rappresentando però il bastione del Cristianesimo occidentale contro la minaccia turca. Soltanto con la sua annessione da parte dellimpero austroungarico e, dopo la pace di Versailles, con l'unione al resto della neonata Jugoslavia, la Dalmazia perse la funzione storica di centro d'italianità, nonostante la presenza di numerosi connazionali e le vestigia di un passato culturale ed artistico strettamente legato alla storia d'Italia53. Per Carelli, il problema era all'epoca costituito dal processo di integrazione che sarebbe dovuto avvenire nella comunità di popoli e di terre poste sotto la giurisdizione imperiale e coloniale della Terza Roma. Lo scopo avrebbe dovuto essere quello di venire integrata con la Croazia e l'Italia.

Tuttavia, la volontà italiana di ottenere un totale controllo dei confini settentrionali e delle coste dell'Adriatico e dei Balcani occidentali, in previsione di trasformare il Mediterraneo in un bacino sotto il dominio di Roma era funzionale al progetto di ottenere anche il controllo sulle materie prime che le altre potenze già possedevano da

$5^{2}$ CARELLI, Gustavo, Dalmazia e $i$ suoi confini, in «Geopolitica», III, 5/1941, pp. 243-249.

53 MONZALI, Luigi, Italiani di Dalmazia. Dal Risorgimento alla Grande Guerra, Firenze, Le Lettere, 2004; MONZALI, Luigi, Italiani di Dalmazia 1914-1924, Firenze, Le Lettere, 2007; CACCAMO, Francesco, Il declino. Gli italiani di Dalmazia dallimpero asburgico alla seconda guerra mondiale, in TRINCHESE, Stefano, CACCAMO, Francesco (a cura di), Adriatico contemporaneo. Rotte e percezioni del mare comune tra Ottocento e Novecento, Milano, Franco Angeli, 2008, pp. 65-121. 
decenni grazie alla loro politica di dominio politico-economico dei possedimenti coloniali, a danno di altre nazioni che, come l'Italia, non avevano un impero ed erano a corto di materie prime.

«Tutta la politica del dopoguerra delle Grandi Democrazie - aveva sottolineato Massi nel 1939 - è volta a consolidare la loro supremazia, ad impedire ogni alterazione di equilibri e ad ostacolare ogni spostamento dei confini»54, in nome dello status quo voluto dopo la Prima guerra mondiale e sancito dal Trattato di Versailles. Per questo, osservava lo studioso triestino, era necessario rivedere gli equilibri internazionali dell'epoca e ripensare il destino dell'Italia all'insegna di una vocazione imperiale che esigeva una ridefinizione degli spazi politici, nella consapevolezza del rapporto che passava tra l'affermazione di una civiltà forte e vitale, cosciente della propria «missione nel mondo», e la sua proiezione spaziale-territoriale.

Ma il sogno imperiale dei geopolitologi italiani ebbe vita breve. Di lì a poco, lepotenze dell'Asse vennero sconfitte dagli Alleati e i presupposti di trasformare l'Italia in una nazione in grado di dominare incontrastata il Mediterraneo, estromettendo dal bacinola presenza di basi e militari dell'impero britannico e francese, venne meno per sempre con la conseguente chiusura, alla fine del 1942, del periodico. Tra le cause anche una carenza di denari per mantenere in vita la rivista.

Le strategie del regime di proseguire lungo la linea tracciata dai corifei della geopolitica italiana con l'ausilio della omonima rivista, vennero riprese ancora per qualche tempo prima che la guerra giungesse all'epilogo. Lo studio della geografia politica avrebbe infatti costituito una branca del neonato Centro Studi per l'Ordine Nuovo, sorto nel maggio del 1943, in linea con i rapporti di alleanza con la Germania nazionalsocialista. Tuttavia, il progetto risaliva almeno ad un anno prima, quando era stato affidato alle cure di Eugenio Coselschi55, che venne nominato presidente.

La sezione geopolitica venne assegnata all'Accademico d'Italia, Giotto Dainelli56, geologo e geografo di fama internazionale, che aderì alla Repubblica Sociale Italiana, e che per la sua perfetta conoscenza del tedesco e delle scienze territoriali manteneva relazioni strette con i colleghi del mondo germanico, i quali commissionavano su ordine delle autorità tedesche l'organizzazione di convegni geografici in territorio

54 MASSI, Ernesto, «Democrazie, colonie e materie prime», cit., p. 18.

55 Sulla figura di Coselschi e la sua attività in seno al regime fascista si vedano i numerosi riferimenti contenuti in CUZZI, Marco, L'Internazionale delle Camicie nere. I CAUR 1933-1939, Milano, Mursia, 2005.

${ }^{5} \mathrm{Su}$ Dainelli si veda il contributo di VEDOVATO, Giuseppe, «Giotto Dainelli, tra scienza e politica», in Rivista di Studi Politici Internazionali, 303, 3/2009, pp. 381-421. 
germanico, come quello che si svolse nel 1942 a Würzburg57 e quello annunciato e mai tenuto, che avrebbe dovuto svolgersi nel 1943 a Vienna.

Il progetto del Centro Studi prevedeva nell'ambito delle scienze territoriali la ripresa delle pubblicazioni della rivista «Geopolitica», mantenendo una sede autonoma nella città di Trieste, ma sotto l'egida del governo di Roma e della sede centrale del Centro Studi situata nella capitale, come osservarono Roletto e Dainelli in uno scambio epistolare avvenuto tra l'11 giugno 1943 e il 24 giugno 1943, conservato nell'Archivio Dainelli della Società Geografica Italiana ${ }^{5}$.

Ma, oramai, la guerra mondiale per le forze dell'Asse volgeva al peggio. La breve stagione della geopolitica italiana terminava definitivamente e senza alternative, legata come era ad una visione imperiale della politica estera e delle scienze territoriali in linea con le prospettive espansioniste nate dai governi liberali italiani e proseguita con l'avvento al potere del Fascismo, che sognava la presenza della nostra Penisola al centro del grande agone mondiale, con un ruolo preminente nel Mediterraneo, come avevano proposto e vagheggiato la gran parte dei padri del Risorgimento, e nel quadro di un rapporto sempre più stretto con l'Africa e le sue immense risorse. Questo doveva essere il ruolo svolto dall'Italia - a detta dei nostri geopolitologi - e tale da costituire un ponte tra il Continente nero e l'Europa, ovvero il centro propulsore e il fulcro della cosiddetta Eurafrica, sinonimo dell'unione geostrategica e geoeconomica dei due continenti.

57 Archivio Centrale dello Stato di Roma, Presidenza del Consiglio dei Ministri, 1940-43, fasc. 14.3/27706, Titolo dell'Affare: Würzburg - Convegno di lavoro del DeutscherGeographentag primavera 1942. Invito a studiosi italiani. I partecipanti al convegno, si sottolineava, che erano tutti iscritti al PNF e furono

- $\quad$ Prof. Riccardi Riccardo - Ordinario di Geografia nella R. Università di Roma.

- $\quad$ Prof. Milone Ferdinando - Ordinario di Geografia economica nella R. Università di Napoli.

- $\quad$ Ecc. Prof. Dainelli Giotto - Ordinario di Geologia nella R. Università di Firenze.

- $\quad$ Prof. Desio Ardito - Ordinario di Geologia nella R. Università di Milano.

- $\quad$ Prof. Migliorini Elio - Libero docente di Geografia nella R. Università di Roma.

- $\quad$ Prof. Toniolo Antonio Renato - Ordinario di Geografia nella R. Università di Bologna.

- $\quad$ Prof. Castiglioni Bruno - Ordinario di Geografia nella R. Università di Pavia.

- $\quad$ Prof. Gortani Michele - Ordinario di Geologia nella R. Università di Bologna.

- $\quad$ Prof. Biasutti Renato - Ordinario di Geografia nella R. Università di Firenze.

- $\quad$ Prof. Morandini Giuseppe - incaricato di Geografia fisica nella R. Università di Roma.

Prof. Fantoli Amilcare - Ministero Africa Italiana.

${ }^{8}$ Archivio Società Geografica Italiana, Fondo Giotto Dainelli, Corrispondenza Dainelli, Roletto, Giorgio, busta 33, fasc. 2352. 


\section{* L'autore}

Andrea Perrone è dottorando in "Beni culturali e Territorio" presso l'Università di Roma "Tor Vergata" con una tesi dedicata alle origini della geopolitica italiana. Ha al suo attivo una serie di articoli e di saggi dedicati alla storia della geopolitica e allo studio analitico di alcune aree di crisi a livello mondiale.

URL: < http://www.studistorici.com/progett/autori/\#Perrone >

\section{Per citare questo articolo:}

PERRONE, Andrea, «Mare nostrum e "Geopolitica". II mito imperiale dei geografi italiani», Diacronie. Studi di Storia Contemporanea : "Se creare è definire", 29/03/2016,

URL:< http://www.studistorici.com/2016/03/29/perrone_numero_25/ >

Diacronie Studi di Storia Contemporanea $\vartheta$ www.diacronie.it

Risorsa digitale indipendente a carattere storiografico. Uscita trimestrale. redazione.diacronie@hotmail.it

Comitato di redazione: Jacopo Bassi - Luca Bufarale - Elisa Grandi - Antonio César Moreno Cantano - Deborah Paci - Fausto Pietrancosta - Alessandro Salvador - Matteo Tomasoni - Luca Zuccolo

Diritti: gli articoli di Diacronie. Studi di Storia Contemporanea sono pubblicati sotto licenza Creative Commons 3.0. Possono essere riprodotti e modificati a patto di indicare eventuali modifiche dei contenuti, di riconoscere la paternità dell'opera e di condividerla allo stesso modo. La citazione di estratti è comunque sempre autorizzata, nei limiti previsti dalla legge. 